\title{
Antimicrobial prescribing pattern in a tertiary care teaching hospital: a pilot study
}

\author{
Bhagyashri D. Rajopadhye ${ }^{1}$, Sonali H. Palkar ${ }^{2 *}$, Vijaya A. Pandit ${ }^{1}$, Priti P. Dhande ${ }^{1}$, \\ Mahadevan Kumar ${ }^{3}$, Sanjay K. Lalwani ${ }^{4}$
}

\begin{abstract}
${ }^{1}$ Department of Pharmacology, ${ }^{2}$ Department of Community Medicine, ${ }^{3}$ Department of Microbiology, ${ }^{4}$ Department of Paediatrics, Bharati Vidyapeeth Medical College, Pune, Maharashtra, India

Received: 19 December 2019

Revised: 07 February 2020

Accepted: 12 February 2020

\section{*Correspondence:}

Dr. Sonali H. Palkar,

Email: palkarsh@gmail.com

Copyright: (C) the author(s), publisher and licensee Medip Academy. This is an open-access article distributed under the terms of the Creative Commons Attribution Non-Commercial License, which permits unrestricted non-commercial use, distribution, and reproduction in any medium, provided the original work is properly cited.
\end{abstract}

\begin{abstract}
Background: The emergence of antimicrobial resistance is a worldwide challenge threatening to negate the gains made by discovery of antimicrobial agents (AMAs). Antimicrobial Stewardship Program (ASP) is an important strategy for ensuring appropriate use of AMAs and controlling emergence of antibiotic resistance. Implementation of ASP must start with assessment of the current state of antimicrobial use. This study was therefore conducted to assess the prevalent prescribing patterns in a tertiary care hospital and identify gaps which warrant corrective intervention.

Methods: This prospective pilot study presents analysis of the first 30 Patients from the medicine ward prescribed at least one antimicrobial agent during the two months of study period. Relevant data was collected in AMA record form. Appropriateness of AMAs was analyzed regarding selection as well as administration protocol as per the hospital antibiotic policy.

Results: Out of the 60 AMAs prescribed to these patients, most commonly prescribed were cephalosporins (63.3\%), anti-amoebics $(26.6 \%)$ and macrolides $(23.3 \%)$. Common diseases involved were urinary tract infection, acute gastroenteritis, sepsis and lower respiratory tract infections. Use of AMAs was found to be appropriate for the indication in $42 \%$, dose in $97 \%$, duration $60 \%$, route $93 \%$ and frequency $90 \%$.

Conclusions: This gap between the appropriate and the actual practice use of AMAs indicates an urgent need of rigorous implementation of ASP in order to avoid emergence of resistance and to conserve the sensitivity to the available AMAs.
\end{abstract}

Keywords: Antibiotic Stewardship Program, Antimicrobial resistance, Irrational prescribing

\section{INTRODUCTION}

Development of antimicrobial agents (AMAs) has been an important milestone in the evolution of modern medicine, enabling elimination of infectious diseases and epidemics and effectively reducing mortality. ${ }^{1}$ However, widespread, irrational and indiscriminate use of antimicrobials has led to emergence of resistance. ${ }^{2,3}$ Antimicrobial resistance is a worldwide challenge, threatening to negate the gains made by the discovery of antimicrobial agents.

Spread of resistant organisms not only hampers cure of infections, but also has adverse impact on health of patients who have never been exposed to them. As reported by the Centre for Disease Control and Prevention (CDC), more than two million people are primarily infected with antibiotic resistant organisms, resulting in approximately 23,000 deaths annually. ${ }^{4}$ 
In India, infectious diseases are still very common and use of AMAs is very high (24-64\%). ${ }^{5}$ In tertiary care hospitals, where patients get admitted for serious illnesses, infection is an everyday problem, making use of antimicrobials extremely common. Tertiary care centres have therefore become the focal points for emergence and spread of resistant pathogens. ${ }^{6,7}$ Compared to the rapid development of resistance, development of new AMAs is a very cost and effort intensive task, with only few new drugs in the pipeline today. Thus, the available AMAs are a precious resource for the whole mankind and must be used judiciously. ${ }^{8,9}$ This makes it of paramount importance to ensure rational use of AMAs and prevent unnecessary and/ or inappropriate use of antimicrobials which can lead to the development of resistance. ${ }^{10}$

Antimicrobial Stewardship Program (ASP) is one of the important strategies for ensuring appropriate use of AMAs and for controlling the emergence of antimicrobial resistance. ${ }^{11}$ Awareness of the need for such a strategy, particularly in the Tertiary Care hospitals, is fortunately increasing, with actual implementation in many such set ups. $^{12,13}$ According to the Deming Cycle or Plan-DoCheck-Act Cycle, planning is the first step of any quality improvement programme. ${ }^{14}$ Prerequisite for planning any improvement is the understanding of the existing situation.

The present study was therefore undertaken to understand the prevalent antimicrobial prescription pattern in a tertiary care teaching hospital embarking on implementation of ASP and to identify gaps in prevalent practices which can be corrected by remedial measures for quality improvement.

\section{METHODS}

After the approval of institutional ethics committee (BVDUMC/IEC/30 dated 14/05/2019), this prospective observational study was conducted at Bharati Vidyapeeth (Deemed to be University) Medical College and Hospital, Pune (Maharashtra). The pilot part of this study included first 30 patients admitted in Medicine ward receiving at least one antimicrobial agent. Data of these patients could be collected in two months, June and July 2019.

Antimicrobial drugs used in these patients was recorded from admission till discharge. An "AMA record form" was prepared for the collection of data, having following components:

- Patient details- demographic as well as diagnostic details

- Drug details- antimicrobial agent prescribed with its dose, route, duration; reason for starting AMA (empirically/ definitive); discharge medication.

- Lab details- relevant investigations for confirming diagnosis- like X- ray chest, TLC etc.; details of culture sensitivity report, if sent.
As a prelude to initiation of ASP, institutional guidelines for use of AMAs were already formulated (Hospital Antibiotic Policy- HAP). ${ }^{15}$ Prescribed AMAs were analysed for appropriateness using these guidelines. Selection of antibiotic was considered appropriate (rational) if it was as per hospital antibiotic policy or a reasonable alternative thereof. Assessment of the rationality of dose, route, duration and frequency of administration of antimicrobial agents was also based on adherence to hospital antibiotic policy.

Change in AMA after culture and sensitivity report, if any, was also assessed.

\section{Analysis of data}

Statistical analysis was conducted using a statistical package for Social Sciences (SPSS) version 25.0.

\section{RESULTS}

\section{Demographic details}

Age range of the study participants was between 20 to 88 years, (average 51.4 years), and $40 \%$ of them were male.

\section{Length of stay}

Hospitalization period ranged from 2-13 days with average length of stay 7.1 days.

\section{Culture sensitivity}

Culture sensitivity tests were performed for $16(54 \%)$ patients, of which, reports of only 14 could be traced. For two patients who received AMA for surgical prophylaxis, culture and sensitivity was not indicated. Importantly, in 12 patients, where such testing was indicated, AMA treatment was initiated without performing culture and sensitivity testing (Table 1).

Table 1: Culture sensitivity tests $(n=30)$.

\begin{tabular}{|llll|}
\hline $\begin{array}{l}\text { Reports } \\
\text { found }\end{array}$ & $\begin{array}{l}\text { Reports not } \\
\text { found }\end{array}$ & $\begin{array}{l}\text { not } \\
\text { applicable }\end{array}$ & $\begin{array}{l}\text { Not } \\
\text { done }\end{array}$ \\
\hline 14 & 2 & 2 & 12 \\
\hline $47 \%$ & $7 \%$ & $7 \%$ & $40 \%$ \\
\hline
\end{tabular}

Total tests done $(\mathrm{n}=16)$.

\section{AMA selection pattern}

AMAs were prescribed either empirically (70\%) or definitively (30\%). Amongst $70 \%$ AMAs started empirically, $40 \%$ were stopped abruptly without any explanatory note (Figure 1 ).

\section{Number of AMAs prescribed per patient}

The range of antibiotics prescribed per patient was 1-5 with an average of 1.9 . Out of 30 patients, $33.3 \%$ received 
one antibiotic, $40 \%$ patients received two antibiotics, $23.3 \%$ patients received three antibiotics and $3.3 \%$ patients received five antibiotics.

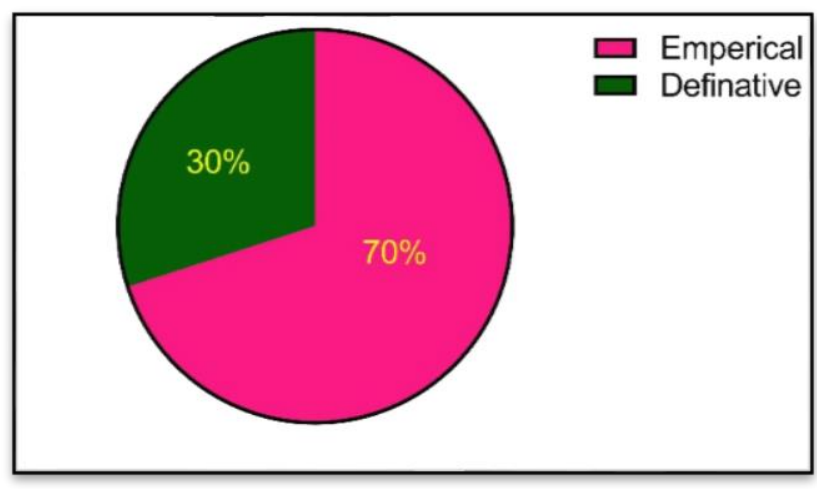

Figure 1: Selection of antimicrobial agents.

\section{Route of administration}

$70 \%$ antibiotics were administered intravenously, $28.4 \%$ were administered by oral route and $1.6 \%$ by ocular instillation. None of the patients was on I.V. infusion.

\section{Infections treated with AMAs}

Most common Infection for which AMAs were prescribed was lower respiratory tract infection (LRTI) (36\%), followed by acute gastroenteritis (AGE) (13\%) and urinary tract infection (UTI) both upper/lower (10\%).

Table 2: Commonly prescribed antimicrobial agents $(n=30)$.

\begin{tabular}{|lll|}
\hline Antimicrobial agents & No. of cases & $\%$ \\
\hline Ceftriaxone & 19 & 63.3 \\
\hline Metronidazole & 8 & 26.6 \\
\hline Azithromycin & 7 & 23.3 \\
\hline Quinolones & 6 & 20 \\
\hline Piperacilin -tazobactum & 7 & 23.3 \\
\hline Amoxicillin-clavulanic acid & 4 & 13.3 \\
\hline
\end{tabular}

\section{Antibiotics prescribed}

Total 60 AMAs were prescribed for the 30 study participants. Most commonly prescribed were cephalosporins- $(70 \%)$, of which ceftriaxone was maximally used $(63.3 \%)$. Next in line were metronidazole (26.6\%), azithromycin (23.3\%) and quinolones (20\%). Fixed dose combinations (FDCs) contributed $36.6 \%$ of antibiotics prescribed. Amongst FDCs, penicillins were most commonly used (40\%), of which piperacilin tazobactum contributed $23.3 \%$, followed by amoxicillinclavulanic acid $(13.3 \%)$. Ceftriaxone was commonly used parenteral AMA $(63 \%)$ (Table 2).
High end AMAs like linezolid, colistin or meropenem were prescribed to less than $3.5 \%$ patients and only as a definitive treatment after culture sensitivity tests.

\section{Assessment of rationality}

As far as rationality of selection of AMAs was concerned, $42 \%$ prescriptions were found to be rational. Almost equal, i.e. $40 \%$, were irrational, while for $18 \%$ of antibiotics prescribed, rationality could not be assessed due to inadequate information (Figure 2).

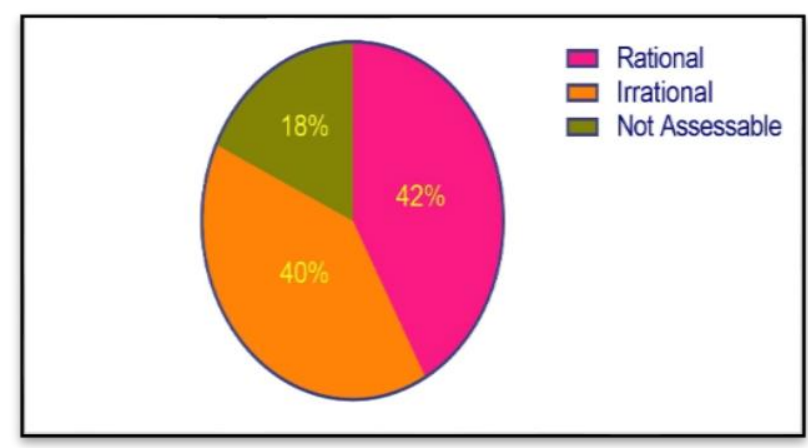

Figure 2: Rationality of selection of antimicrobial agents.

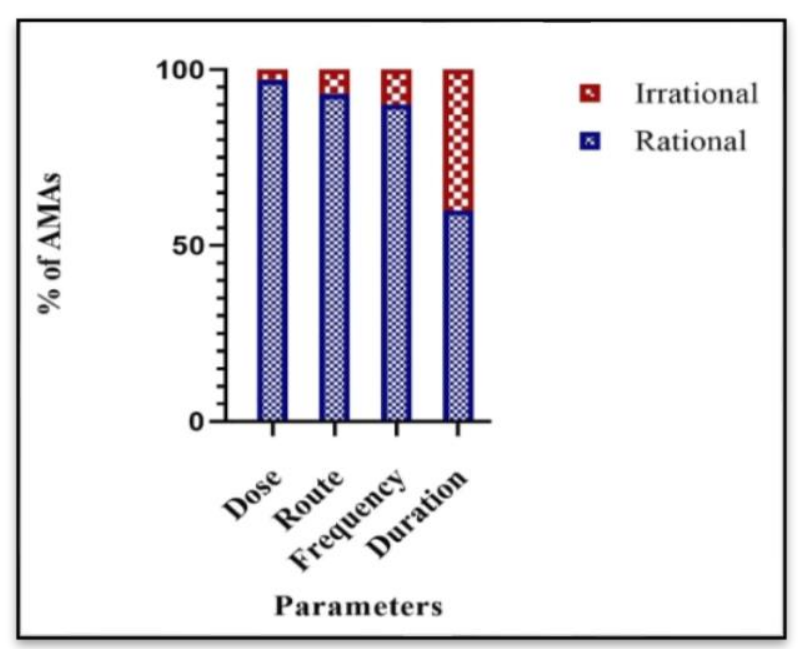

Figure 3: Rationality of administration of antimicrobial agents.

\section{Assessment of administration of AMAs}

Figure 3 showed that dose (97\%), frequency of administration $(90 \%)$ and route $(93 \%)$ were rational. However, duration was appropriate in only $60 \%$ AMAs.

\section{Drug interactions and compatibility}

No drug interactions as well as physical or chemical incompatibility was observed amongst the antibiotics prescribed. 


\section{DISCUSSION}

Rational use of drugs implies that patients receive medications appropriate to their clinical needs, in doses that meet their own individual requirements, for an adequate period of time, at the lowest cost to them and their community. ${ }^{16}$

Analysis of the data of thirty patients receiving at least one AMA has revealed substantial element of irrational prescribing. Out of the $70 \%$ AMAs started empirically, the choice was appropriate for the indication in only $40 \%$. Rajallingam et al who also evaluated rationality of use of AMA in a private tertiary care teaching hospital found that the choice of antibiotics was appropriate only in $61 \%$ cases. $^{17}$

We found Ceftriaxone to be excessively used (63\%) in our patients (Table 2). Rajallingam et al also reported Ceftriaxone to be the most commonly used antibiotic at the private tertiary care teaching hospital at Coimbatore, although the magnitude of use $(15.4 \%)$ was much less than in our study. Shirin et al have also reported Cephalosporin group as the most commonly prescribed group, though it was prescribed only in $22 \%$ cases studied. ${ }^{18}$ Wide spectrum of action and less adverse effects was considered to be the probable reason prompting its excessive use. ${ }^{19}$

Table 3: Examples for irrational (inappropriate) selection of antimicrobial agents.

\begin{tabular}{|lll|}
\hline $\begin{array}{l}\text { Antimicrobial } \\
\text { agents used }\end{array}$ & $\begin{array}{l}\text { Clinical } \\
\text { condition }\end{array}$ & Inappropriateness \\
\hline Ceftriaxone & $\begin{array}{l}\text { Acute } \\
\text { gastroenteritis }\end{array}$ & AMA not required \\
\hline Ceftriaxone & $\begin{array}{l}\text { Upper } \\
\text { respiratory } \\
\text { tract infection }\end{array}$ & AMA not required \\
\hline Ceftriaxone & Dengue & AMA not required \\
\hline Ceftriaxone & Viral Fever & AMA not required \\
\hline $\begin{array}{l}\text { Amoxicillin- } \\
\text { clavulanic } \\
\text { acid }\end{array}$ & $\begin{array}{l}\text { Cough with } \\
\text { expectoration }\end{array}$ & AMA not required \\
\hline Amikacin & $\begin{array}{l}\text { Lower Urinary } \\
\text { Tract Infection }\end{array}$ & Wrong AMA \\
\hline
\end{tabular}

In this study, FDCs contributed $36.6 \%$ of antibiotics prescribed. Another study in a tertiary care hospital in Trivandrum reported only $17 \%$ contribution of FDCs. ${ }^{20}$

Thus, in present study, major irrationality was in the selection of AMAs (40\%). Table 3 depicts some of the instances of inappropriate selection of AMA, indicating disregard or unawareness of the Hospital Antibiotic Policy. Inappropriateness was also present for the duration of AMA use $(40 \%)$. Only $10 \%$ prescriptions were inappropriate for the dose, frequency and route of administration.
Another important factor which may have confounded our analysis was inadequate documentation by resident doctors. For example, cases diagnosed as LRTI did not record any supportive investigations like $X$ ray chest or culture sensitivity of sputum. Thus, inability to assess the rationality of $18 \%$ of prescriptions was due to lack of adequate information for the confirmation of diagnosis or giving the reason for initiation or termination of the AMA.

Thus, we could identify gaps in prescription practices. There was non-compliance with hospital antibiotic policy for selecting AMAs in $40 \%$ cases. Records maintained were incomplete in terms of basis for initiation and/or change in AMAs, due to which analysis of these prescriptions could not be done. Large scale use of ceftriaxone was also a big concern.

Inadequacies in data collection also got highlighted in this study. Data collection team plays an important role in ASP and all the conclusions essentially depend on the information collected. The information was collected by data collection form and then entered on the excel sheet. It was found that errors had happened during transferring the data onto the excel sheets. Though digitization of data entry remains the best option to overcome this flaw, such a system is not yet available. So, in the present scenario, there was the need for reinforcing the training of the data collecting team. Need was also felt to improvise data collection form for proper documentation.

In a nutshell, this pilot study revealed that interventions were needed for prescribers as well as for data collection team. Apart from emphasising the need for proper use of AMAs, training of prescribers on doing and documenting relevant investigations; understanding and implementing HAP and real time scrutiny of AMA prescriptions was required. Training of data collection team was also required.

\section{CONCLUSION}

The emergence of antimicrobial resistance is a worldwide challenge attributed to widespread, irrational and indiscriminate use of antimicrobials. There is an urgent need to control the situation to retain sensitivity to the basic AMAs. Antibiotic Stewardship Programme (ASP), is accepted in the world as an effective measure to ensure rational use of AMAs and prevent emergence of resistance.

Before implementing ASP, it was required to understand the existing practises of use of AMAs. A study was planned for such an evaluation. To begin with, a pilot study was carried out to ascertain the efficacy of the data collecting tool and also to get an idea about the use of AMAs in the hospital.

The pilot study revealed that on prescriber's side, gross irrationality existed in selecting AMA and adhering to the 
duration of the therapy. Some modification in the data collecting form was also required for the accuracy and the completeness of the information. Training of the data collecting team was also required.

\section{ACKNOWLEDGEMENTS}

Authors would like to thank Clinical Pharmacy Interns of Bharati Vidyapeeth Medical College and Hospital, Pune for their contribution in data collection.

\section{Funding: No funding sources}

Conflict of interest: None declared

Ethical approval: The study was approved by the Institutional Ethics Committee of Bharati Vidyapeeth (Deemed to be University) Medical College, Pune, Maharashtra, India. (BVDUMC/IEC/30 dated 14/05/2019).

\section{REFERENCES}

1. Adiveni T, Chandra DS, Sundresh NJ, Kumar BA, Padmini P, Haritha K. Analysing The Rationality of Antibiotics In Comparing The Different Departments In Rmmch. J Biomed Pharmaceut Res. 2013;2(3):746.

2. Spellberg B, Guidos R, Gilbert D, Bradley J, Boucher HW, Scheld WM, et al. The epidemic of antibioticresistant infections: a call to action for the medical community from the Infectious Diseases Society of America. Clini Infect Dis. 2008;46(2):155-64.

3. Bbosa GS, Geoff W, Kyegombe DB, Ogwal-Okeng J. Effects of intervention measures on irrational antibiotics/antibacterial drug use in developing countries: A Systematic Review. Health 6 . 2014;6(2):171-87.

4. Centres for Disease Control and Prevention (CDC). Core elements of Hospital Antibiotic Stewardship Programs. Atlanta, GA: U.S. Department of Health and Human Services, CDC; 2014. Available at: http://www.cdc.gov/getsmart/healthcare/pdfs/coreelements.html. Accessed 12 April 2019

5. Vanitha M, Vineela M, Benjamin RK. Prescribing pattern of antibiotics in patients attending ENT OPD in a tertiary care hospital. IOSR-JDMS. 2017;16:303 .

6. Kulshrestha S, Aggrawal K. Survey of pattern of antimicrobial use in teaching hospital. Ind $\mathbf{J}$ Pharmacol. 1984;16(1):395.

7. Kunin C. Rational use of antibiotics. WHO Drug Info. 1990;4(1):7.

8. Ventola CL. The antibiotic resistance crisis: part 1: causes and threats. Pharma Therapeut. 2015;40(4):277.
9. Fair RJ, Tor Y. Antibiotics and bacterial resistance in the 21st century. Perspectives Med Chem. 2014;6:PMC-S14459.

10. Upadhyay DK, Palaian S, Shankar PR, Mishra P. Rational Drug Prescribing and Dispensing in Tertiary Care Teaching Hospital of Western Nepal. J Institute Med. 2008;30:233-8.

11. Diaz Granados CA. Prospective audit for antimicrobial stewardship in intensive care: impact on resistance and clinical outcomes. Am J Infect Control. 2012;40(6):526-9.

12. Elligsen M, Walker SA, Pinto R, Simor A, Mubareka $\mathrm{S}$, Rachlis A, et al. Audit and feedback to reduce broad-spectrum antibiotic use among intensive care unit patients a controlled interrupted time series analysis. Infect Control Hospital Epidemiol. 2012;33(4):354-61.

13. Singh S, Menon VP, Mohamed ZU, Kumar VA, Nampoothiri V, Sudhir S, et al. Implementation and impact of an antimicrobial stewardship program at a tertiary care center in South India. In Open Forum Infect Dis. 2018;6(4):290.

14. Realyvásquez-Vargas A, Arredondo-Soto K, Carrillo-Gutiérrez T, Ravelo G. Applying the PlanDo-Check-Act (PDCA) Cycle to Reduce the Defects in the Manufacturing Industry: A Case Study. Appl Sci. 2018; 8(11):2181.

15. Tolpadi A, Purandare B, Shah J, Iyer S (eds). Antibiotic Policy. 3rd edition. Pune, India: Bharati Hospital \& Research Centre; 2017: 1-52.

16. Farhan AK, Vinod KS, Sanjeevsharma, Preeti SA. Prospective Study on the Antimicrobial Usage in the Medicine Department of a Tertiary Care Teaching Hospital J Clini Diagnos Res. 2013;7(7):1343-6.

17. Rajalingam B, Alex AS, Godwin A, Cherian C, Cyriac C. Assessment of rational use of antibiotics in a private tertiary care teaching hospital. Ind $\mathrm{J}$ Pharma Pract. 2016; 9(1):14-8.

18. Jokandan SS, Jha DK. A study of prescribing pattern of antibiotic in a tertiary care hospital - an observational study. Int J Pharm Sci Res 2019;10(5):2285-89.

19. Lee H, Jung D, Yeom JS, Son JS, Jung SI, Kim YS, et al. Evaluation of ceftriaxone utilization at multicenter study. Korean J Int Med. 2009;24(4):374.

20. Remesh A, Salim S, Gayathri AM, Nair U, Retnavally KG. Antibiotics prescribing pattern in the in-patient departments of a tertiary care hospital. Arch Pharma Pract. 2013;4(2):71.

Cite this article as: Rajopadhye BD, Palkar SH, Pandit VA, Dhande PP, Kumar M, Lalwani SK. Antimicrobial prescribing pattern in a tertiary care teaching hospital: a pilot study. Int J Basic Clin Pharmacol 2020;9:465-9. 